\title{
An accessory flexor digiti minimi brevis or flexor digiti minimi longus?*
}

\author{
Ceren Günenç Beşer, Mustafa F. Sargon, Burcu Erçakmak \\ Department of Anatomy, Faculty of Medicine, Hacettepe University, Ankara, Turkey
}

\begin{abstract}
During routine gross anatomical dissection of the right forearm and hand region of a 61 year old male cadaver, an unusual muscle originating from the tendon of palmaris longus muscle was observed. This muscle originated $3.1 \mathrm{~cm}$ proximal to the proximal end of flexor retinaculum and coursed superficial to the retinaculum to reach the hypothenar region. In the hypothenar region, $5.2 \mathrm{~cm}$ distal to the proximal end of flexor retinaculum, the muscle fibers became tendinous and this tendon was inserted to the medial side of the base of fifth proximal phalanx. In this cadaver, muscle fibers of flexor digiti minimi brevis was also inserted to the medial side of this variant tendon in a unipennate manner and reached to its final insertion. Our case is unique and the possibility of the presence of such an anomalous huge muscle must always be remembered by the clinicians when a patient has findings of ulnar nerve compression syndrome or hypovascularity symptoms in the hand area due to the compression of ulnar artery.
\end{abstract}

Keywords: accessory flexor digiti minimi brevis muscle; anatomy; flexor digiti minimi longus muscle; human cadaver variation Anatomy 2015;9(1):45-47 @2015 Turkish Society of Anatomy and Clinical Anatomy (TSACA)

\section{Introduction}

The muscles of the limbs are started to develop in the seventh week as a condensation of mesenchyme beside the base of the limb buds. The mesenchyme is derived from dorsolateral cells of the somites. When these limb buds elongate, the muscle tissue splits into flexor and extensor components. At first, the muscles of the limbs are segmental, and then they fuse. The upper limb buds lie opposite the lower five cervical and upper two thoracic segments. ${ }^{[1]}$

In the literature, there are many studies related to the variations of the hypothenar muscles. These include fusion, absence or multiplication type of variations frequently. ${ }^{[2-4]}$ Some of these hypothenar muscle variations may cause ulnar nerve compression syndromes and therefore have a great clinical importance for the clinicians. ${ }^{[5-7]} \mathrm{In}$ this case, we report an extremely rare variation of one of the muscles of hypothenar region and rename it according to its anatomical localization.

\section{Case Report}

During the routine gross anatomical dissection of the right forearm and hand region of a 61 year old male cadaver, an unusual muscle originating from the tendon of palmaris longus muscle was observed. This muscle was originated $3.1 \mathrm{~cm}$ proximal to the proximal end of flexor retinaculum and coursed superficial to the retinaculum to reach the hypothenar region. In the hypothenar region, $5.2 \mathrm{~cm}$ distal to the proximal end of flexor retinaculum, the muscle fibers became tendinous, and this tendon was inserted to the medial side of the base of fifth proximal phalanx. In this cadaver, the muscle fibers of the flexor digiti minimi brevis muscle was also inserted to the medial side of this variant tendon in a unipennate manner and reached its final insertion (Figures 1 and 2). The left forearm and hand region of the cadaver was normal in every aspect.

\section{Discussion}

Variations of the hypothenar muscles are common and they may cause to vascular and nerve compression syn-

*This study was presented as an oral presentation in 9th EACA Congress, 5-8 September 2007, Prague, Czech Republic. 


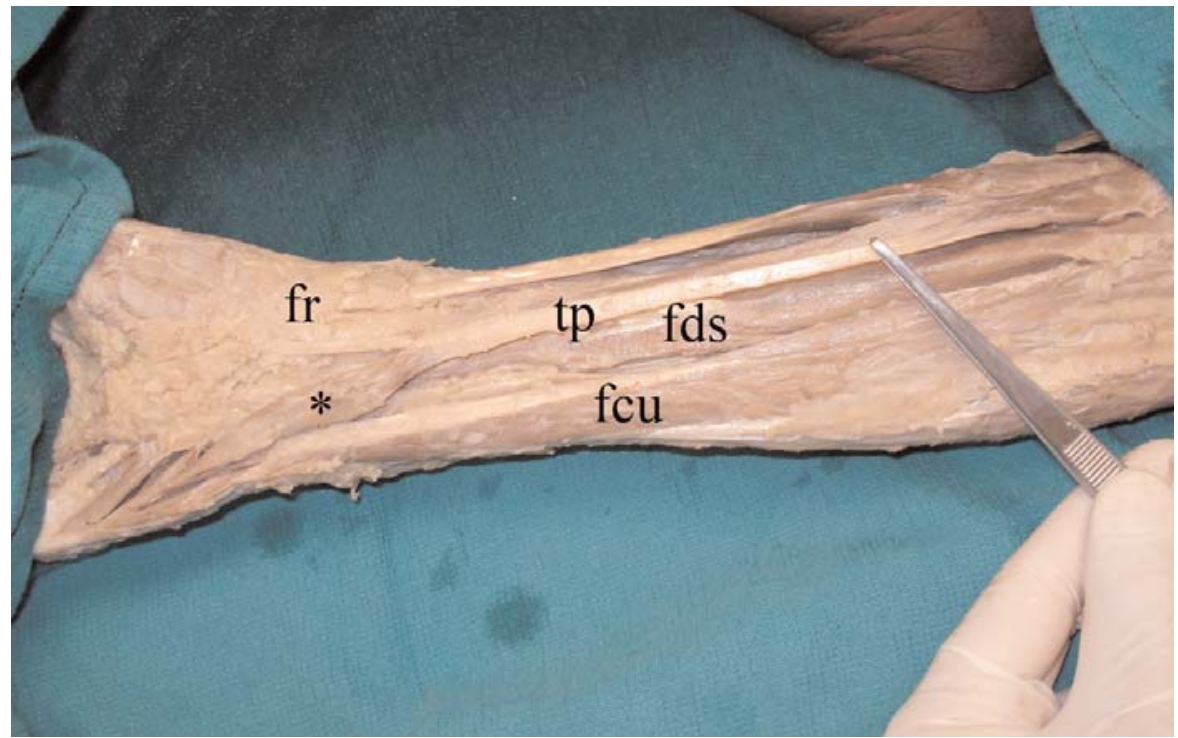

Figure 1. The accessory flexor digiti minimi brevis muscle $\left(^{*}\right)$ in a cadaver. fcu: flexor carpi ulnaris muscle; fds: flexor digitorum superficialis muscle; fr: flexor retinaculum; tp: tendon of palmaris longus muscle. dromes depending upon their anatomical localization. Saadeh and Bergman described an accessory flexor (opponens) digiti minimi muscle with an origin from the antebrachial fascia. ${ }^{[2]}$ Soldado-Carrera et al. found an accessory belly of the abductor digiti minimi muscle arising from the tendon of the palmaris longus muscle in the lower third of the forearm. ${ }^{[3]}$ Wahba et al. observed an anomalous accessory flexor digiti minimi profundus muscle. The identified muscle originated from the intercompartmental septum on the medial side of the forearm just proximal to the wrist joint and inserted on the proximal phalanx of the fifth digit. ${ }^{[6]}$ Bakinde et al. described an accessory muscle which originated from the tendon of flexor carpi radialis, passing palmar to the flexor retinaculum and the ulnar nerve through Guyon's canal. This muscle inserted about $1 \mathrm{~cm}$ proximal to the insertion of abductor digiti minimi muscle and also received fibers from this muscle. ${ }^{[4]}$ De Smet studied the median and ulnar nerve compressions at the wrist region caused by anomalous muscles. According to the author, the variations of palmaris longus, abductor digiti minimi group and flexor carpi ulnaris may cause idiopathic ulnar tunnel syndrome. ${ }^{[5]} \mathrm{Uz}$ et al. found a double palmaris longus muscle and an accessory flexor digiti minimi brevis muscle at the same hand. This accesory muscle originated from the flexor retinaculum and attached to the tendon of the flexor digiti minimi brevis. ${ }^{[8]}$ Bergman et al.

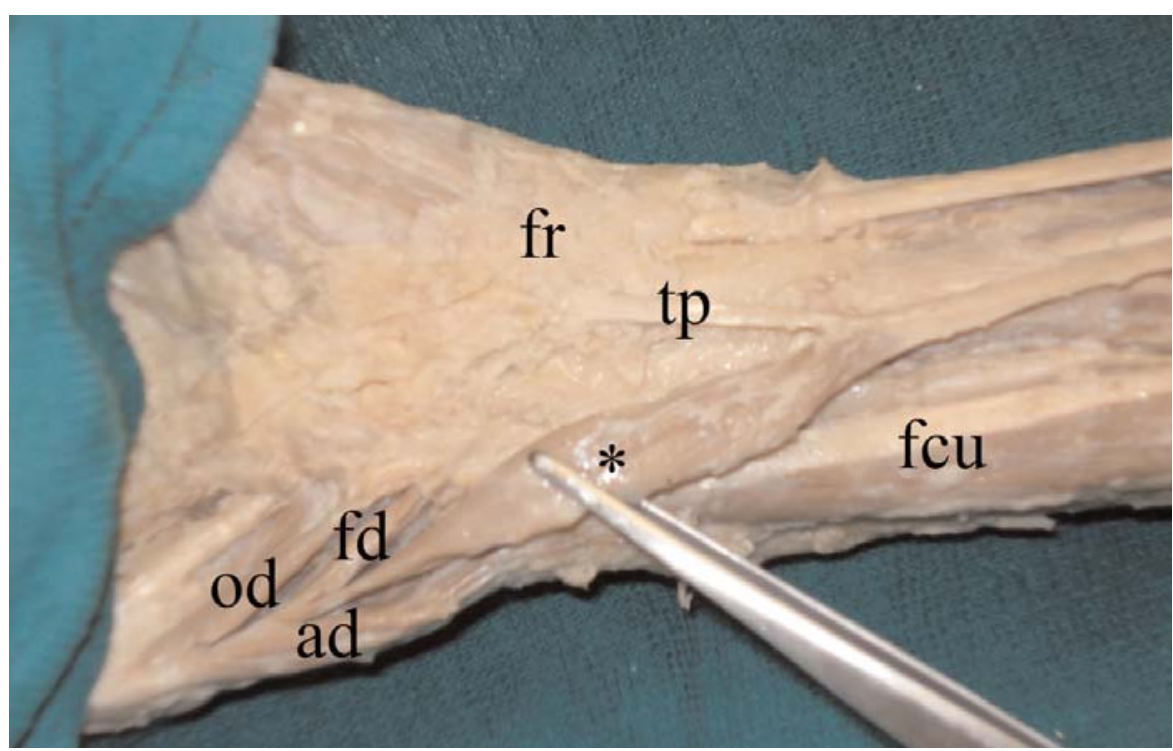

Anatomy • Volume 9 / Issue 1 / April 2015
Figure 2. The accessory flexor digiti minimi brevis muscle $\left(^{*}\right)$ and hypothenar muscles. ad: abductor digiti minimi muscle; fd: flexor digiti minimi brevis muscle; fr: flexor retinaculum; od: opponens digiti minimi muscle; tp: tendon of palmaris longus muscle. 
mentioned that the tendon of palmaris longus muscle may give rise to an additional muscle, accessorius ad flexorem digiti minimi, which usually inserts on the body and head of the fifth metacarpal between abductor digiti minimi and flexor digiti minimi brevis muscles. It has been reported that an accessory muscle may have two bellies, one arising from the tendon of flexor carpi ulnaris and the other arising from the tendon of palmaris longus. ${ }^{[9]}$ Greiner found an anomalous extra muscle that originated from the distal medial aspect of the ulnar tuberosity and passed through the carpal tunnel. The tendon of this muscle fused with the flexor digitorum profundus and inserted on the base of the distal phalanx of the fifth digit. ${ }^{[10]}$ Cope and Little reported a variation of the flexor digiti minimi brevis with connections with abductor digiti minimi and palmaris longus. This muscle had proximal attachments on the hamate bone and flexor retinaculum, and a distal attachment at the anteromedial surface of the proximal phalanx of the fifth digit. It had a $53 \mathrm{~mm}$ muscular attachment to the medial aspect of palmaris longus. ${ }^{[1]}$

In the literature, there is only one case report which resembles our case. In this case, Morrison found a muscle taking origin from the tendon of the palmaris longus about 2 inches above the wrist; it passed as a small somewhat flattened muscle belly, distally and medially, into the palm of the hand. About one-quarter of the distance from the pisiform to the base of the fifth metacarpal, the muscle belly tapered off into a delicate tendon which ran on between the abductor digiti minimi and the opponens. With both muscles it finally blended, receiving a common insertion into the base of the proximal phalanx of the little finger on its medial side ${ }^{[12]}$ However, in our case, the variant muscle originated more close to the wrist joint and additionally, we had a very normal flexor digiti minimi brevis muscle in its appropriate anatomical localization (Figures 1, 2). Therefore, our case is unique and shows a difference from the described case. Morrison doubtlessly defined the variant muscle as an additional belly of the palmaris longus muscle. According to the author, this muscle was digastric and had insertions into the thumb or little finger. However in our case, depending upon to the insertion and function of the variant muscle, we included it to the group of hypothenar muscles. Secondly, because of its same insertion to the little finger with the flexor digiti minimi brevis and its similar function, we suggest to name this very unusual and long hypothenar muscle as the flexor digiti minimi longus or accessory flexor digiti minimi brevis in the case of its presence.

The possibility of the presence of such an anomalous huge muscle must always be remembered by the clinicians when a patient has findings of ulnar nerve compression syndrome or hypovascularity symptoms in the hand area due to the compression of ulnar artery.

\section{References}

1. Sadler TW. Langman's medical embryology. 10th ed. Philadelphia (PA): Lippincott Williams and Wilkins; 2006. p. 146-7.

2. Saadeh FA, Bergman RA. An unusual accessory flexor (opponens) digiti minimi muscle. Anat Anz 1988;165:327-9.

3. Soldado-Carrera F, Vilar-Coromina N, Rodriguez- Baeza A. An accessory belly of the abductor digiti minimi muscle: a case report and embryologic aspects. Surg Radiol Anat 2000;22:51-4.

4. Bakinde N, Yotovski P, Voigt T, Rager G. Accessory muscle in the hypothenar region: a functional approach. Ann Anat 2005;187: 149-52.

5. De Smet L. Median and ulnar nerve compression at the wrist caused by anomalous muscles. Acta Orthop Belg 2002;68:431-8.

6. Wahba MY, Singh GD, Lozanoff S. An anomalous accessory flexor digiti minimi profundus muscle: A case story. Clin Anat 1998; 11:55-9.

7. Madhavi C, Holla SJ. Anomalous flexor digiti minimi brevis in Guyon's Canal. Clin Anat 2003;16:340-3.

8. Uz A, Tagil SM. The case of unilateral double m. palmaris longus with bilateral $\mathrm{m}$. flexor digiti minimi brevis accessorius. Ankara Üniversitesi Tıp Fakültesi Mecmuası 2002;55:167-70. [Turkish]

9. Bergman RA, Afifi AK, Miyauchi R. Illustrated encyclopedia of human anatomic variation. Opus I: muscular system [Internet]. [Revised on March 24, 2015] Available from: http://www.anatomyatlases.org/AnatomicVariants/AnatomyHP.shtml

10. Greiner TM. An additional flexor of the fifth digit: flexor digiti minimi longus. Clin Anat 2008;21:792-3.

11. Cope JM, Little T. A robust flexor digiti minimi brevis muscle. International Journal of Anatomical Variations 2013; 6:216-7.

12. Morrison JT. A palmaris longus muscle with a reversed belly, forming an accessory flexor muscle of the little finger. J Anat Physiol 1916;50:324-6.

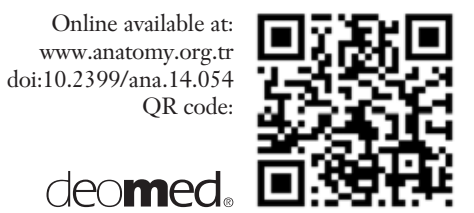

\author{
Correspondence to: Ceren Günenç Beşer, MD \\ Department of Anatomy, Faculty of Medicine, \\ Hacettepe University, 06100, Ankara, Turkey \\ Phone: +90 3123052117 \\ e-mail: crngnc5er@gmail.com
}

Conflict of interest statement: No conflicts declared.

\footnotetext{
This is an open access article distributed under the terms of the Creative Commons Attribution-NonCommercial-NoDerivs 3.0 Unported (CC BY-NCND3.0) Licence (http://creativecommons.org/licenses/by-nc-nd/3.0/) which permits unrestricted noncommercial use, distribution, and reproduction in any medium, provided the original work is properly cited. Please cite this article as: Günenç Beşer C, Sargon MF, Erçakmak B. An accessory flexor digiti minimi brevis or flexor digiti minimi longus? Anatomy 2015;9(1):45-47.
} 